\title{
Laser measured rate equations with various transmission coders for optimum of data transmission error rates
}

\author{
Hazem M. El-Hageen ${ }^{1}$, Aadel M. Alatwi², Ahmed Nabih Zaki Rashed ${ }^{3}$ \\ ${ }^{1}$ Egyptian Nuclear \& Radiological Regulatory Authority, Cairo, Egypt \\ ${ }^{1,2}$ Electrical Engineering Department, Faculty of Engineering, University of Tabuk, Tabuk, Saudi Arabia \\ ${ }^{3}$ Faculty of Electronic Engineering, Menoufia University, Egypt
}

\begin{tabular}{l} 
Article Info \\
\hline Article history: \\
Received Apr 10, 2020 \\
Revised Jun 1, 2020 \\
Accepted Jun 26, 202 \\
\hline Keywords: \\
Data rates optimum \\
Laser measured \\
Rate equations \\
Transmission coder
\end{tabular}

\begin{abstract}
The present study has outlined laser-measured rate equations with various transmission coders for optimum data transmission error rates. Various modulation transmission coders are employed, such as a pulse position modulation coder, a differential pulse intensity modulation coder, and a four band/five band modulation transmission coder, in order to create optimized data rates of up to $40 \mathrm{~GB} / \mathrm{s}$ for a fiber extension length of up to $100 \mathrm{~km}$. This study has emphasized the important role of pulse position modulation transmission coders, which exhibit superior performance in max. $\mathrm{Q}$ parameter and min. data error rates, even for high data rate transmission
\end{abstract}

Copyright $@ 2020$ Institute of Advanced Engineering and Science. All rights reserved.

\section{Corresponding Author:}

Ahmed Nabih Zaki Rashed, Faculty of Electronic Engineering, Menouf, Menoufia University, Egypt E-mail:ahmed_733@yahoo.com

\section{RELATED WORKS}

Heterojunctions juxtapose two different doping or bandgap semiconductor materials [1-3]. They are used in the fabrication of many solid-state devices, including transistors, photodetectors, and solar cells, to name a few. They were first used in 1969-70 to reduce the threshold current density in injection lasers [1-4]. However, a double heterojunction structure was used to reduce the lasing threshold current by a factor of approximately 100. This is one of the effects of the confinement of the resulting carrier, which is expressed by the optical confinement factor [5-7]. Many recent studies have been interested in the application of heterojunctions in vertical-cavity surface-emitting lasers and have investigated their electrical characteristics [8-10]. Typically, heterojunctions are made on either side of a thin active layer, and both carriers and light are confined. This reduces the losses of both, and consequently, the laser oscillation threshold is significantly reduced $[11,12]$. In forward bias, the positive side of the heterojunction structure connects to the positive terminal, and vice versa [13-17]. The heterojunction's structure also reduces the defects in the lattice between the two layers, at which missing or dangling bonds may be formed, such as misfit dislocations or inclusions. These defects lead to nonradiative recombination, which reduces quantum device efficiency [18-21]. Therefore, it is recommended that heterojunctions should not have a lattice parameter mismatch greater than $0.1 \%$ [22-30].

\section{MODEL DESCRIPTION AND RESEARCH METHODS}

The data source generates a stream bit sequence of 10101100 and the bits stream is encoded/configured through different transmission coders and through the non-return to zero code pulse 
generator. These used transmission coders include a pulse position modulation (PPM) transmission coder, a differential pulse intensity modulation (DPIM) transmission coder, and a four band/five band (4B5B) coder. The encoded signal is then forwarded to the light source with a frequency of $1550 \mathrm{~nm}$, a test temperature of $27^{\circ} \mathrm{C}$, and a reference temperature of $20^{\circ} \mathrm{C}$. The encoded bits are used for a transmission coder of two bits per symbol. The VCSEL, which is measured by laser, converts the electrical coded signal to the light signal. The internal modulated signal is then forwarded to the fiber-optic cable length of $100 \mathrm{~km}$.

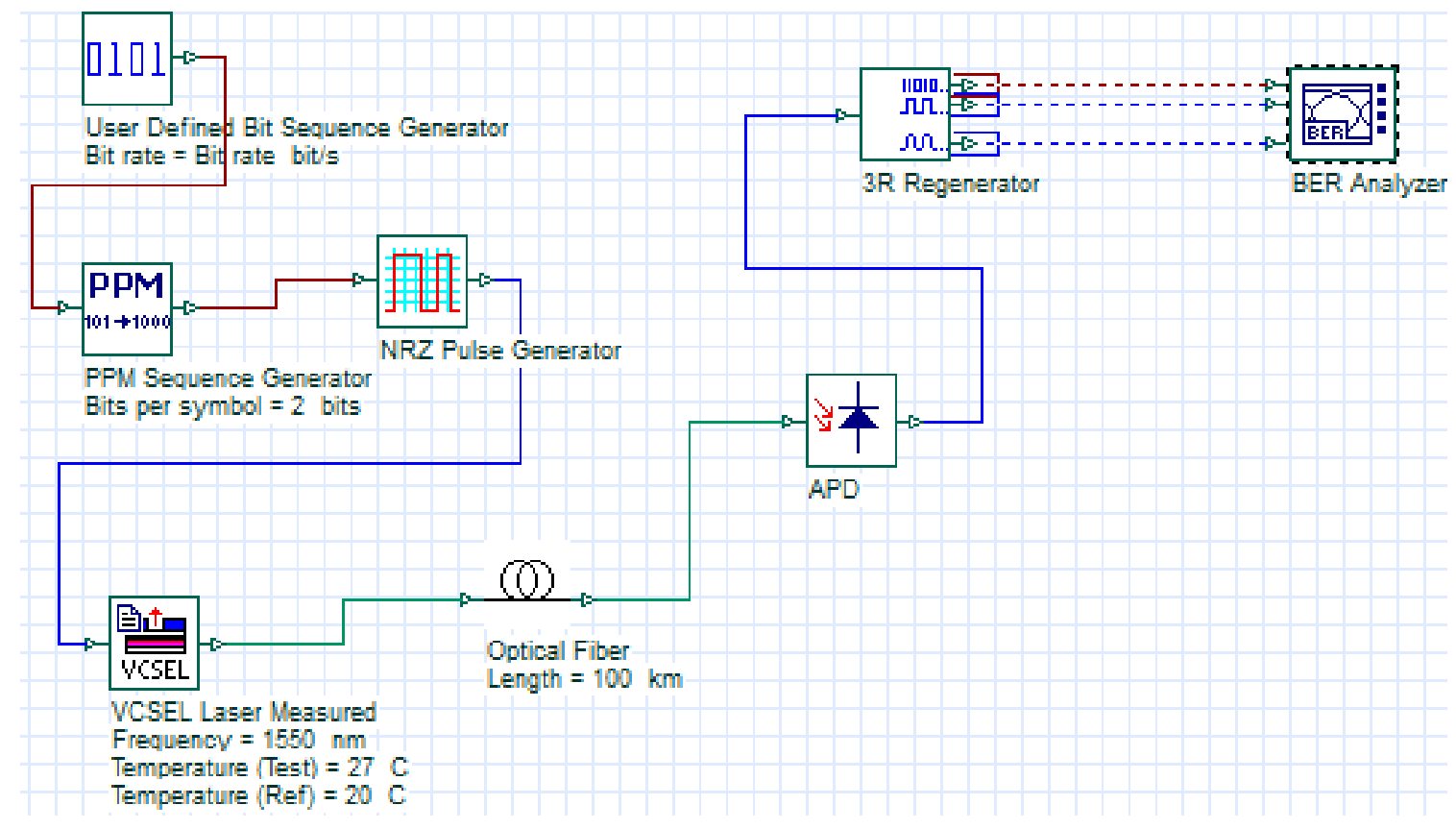

Figure 1. VCSEL measured with the laser model for the pulse coders

The light signal is then directed from the fiber-optic cable to the light avalanche detector in order to convert it to the electrical signal form. The signal is retimed/regenerated/reshaped with three operation generators, and the maximum quality factor coefficient and minimum data rate error are detected through the data error rate analyzer.

\section{PERFORMANCE ANALYSIS WITH DISCUSSIONS}

Figures 2 and 3 indicate the max. Q parameter and the minimum data error rate value estimation based on the pulse position modulation transmission coder. The max. Q parameter achieved a value of 4.05, and the minimum data error rates achieved a value of $2.1 \times 10^{-5}$. Figures 4 and 5 clarify the max. Q parameter value estimation based on the differential pulse intensity modulation (DPIM) transmission coder. The max. Q parameter value achieved a value of 1.784, and the minimum data error rates achieved a value of 0.03198 .

The max. Q parameter and minimum data error rate values were estimated based on a four band/five band modulation transmission coder, as shown in Figures 6 and 7, where the Max. Q parameter achieved a value of 3.16 and the minimum data error rates achieved a value of $7.59 \times 10^{-4}$. Figure 8 shows the relation between the maximum Q coefficient factor parameters, with an available propagation distance range of up to $100 \mathrm{~km}$. The negative effects can be observed in the increasing propagation distance and the signal transmission quality, and the PPM transmission coder has performed better than other transmission coders. 

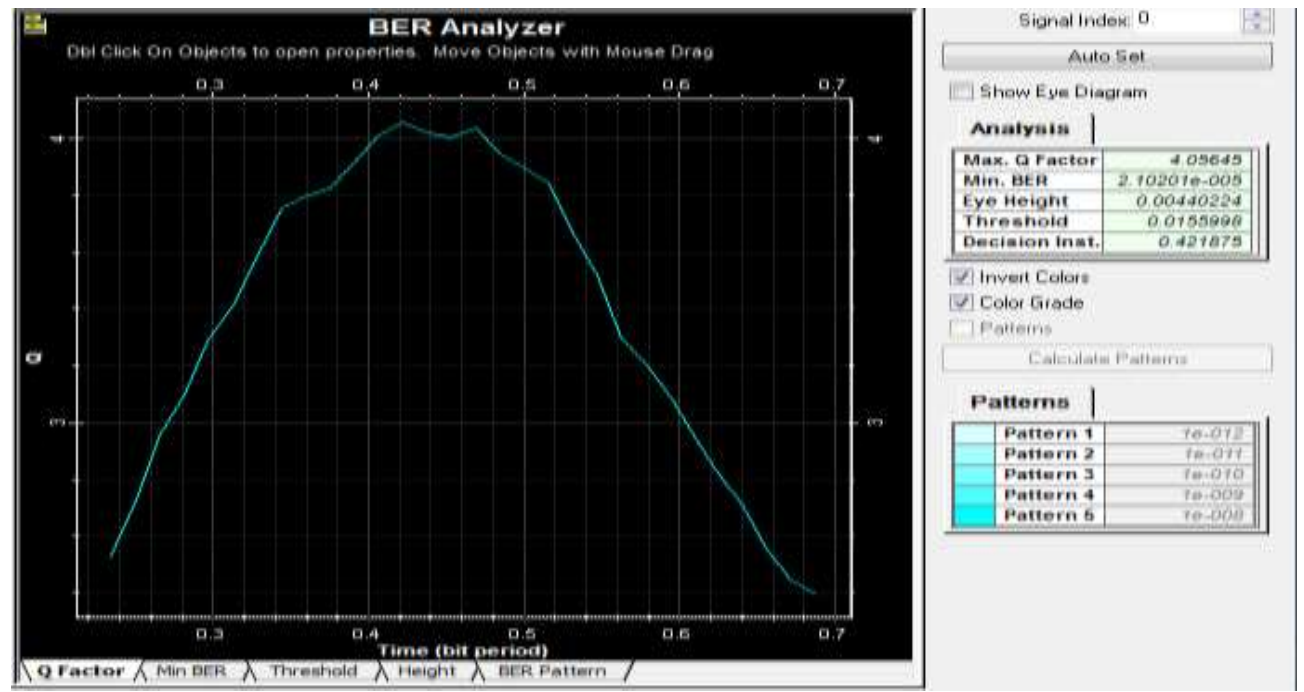

Figure 2. Max. Q parameter value estimation based on the pulse position modulation transmission coder
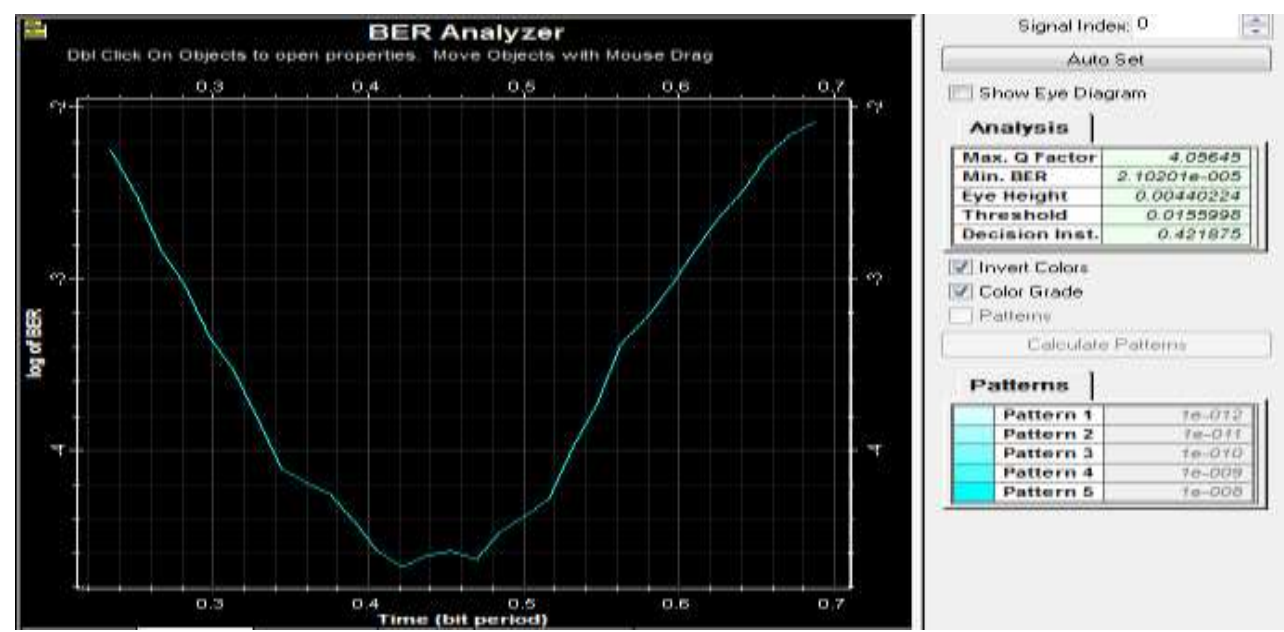

Figure 3. Min. data error rates value estimation based on the pulse position modulation transmission coder
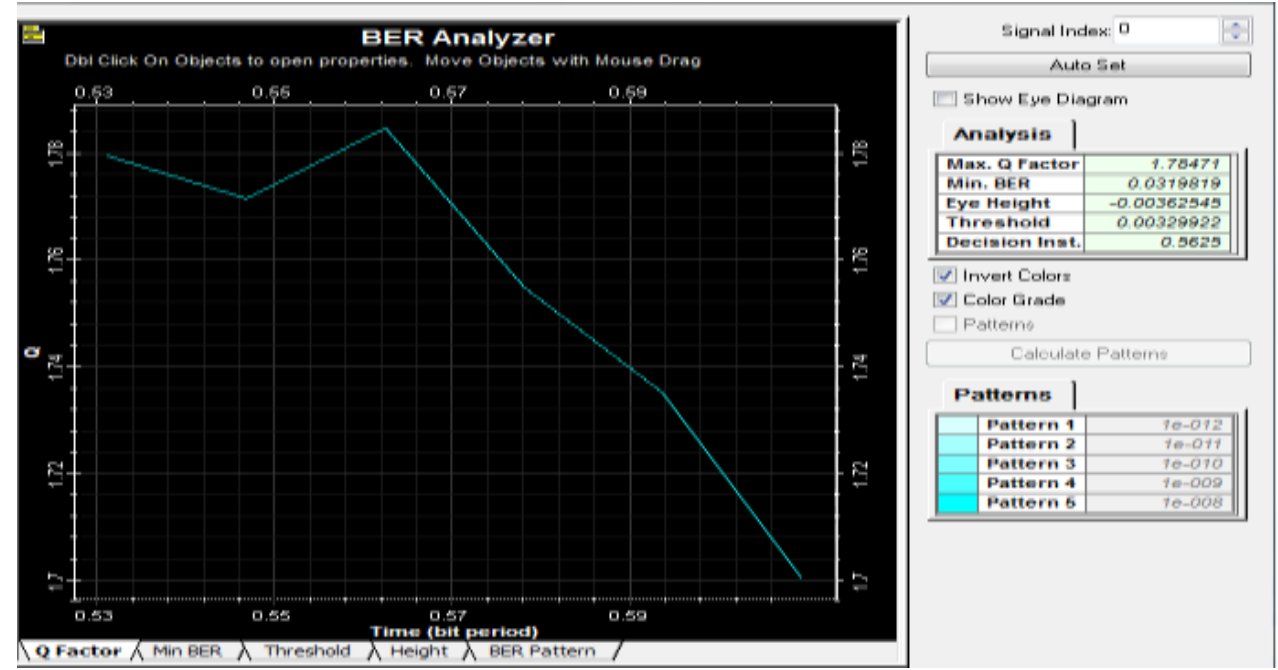

Figure 4. Max. Q parameter value estimation based on the DPIM transmission coder 

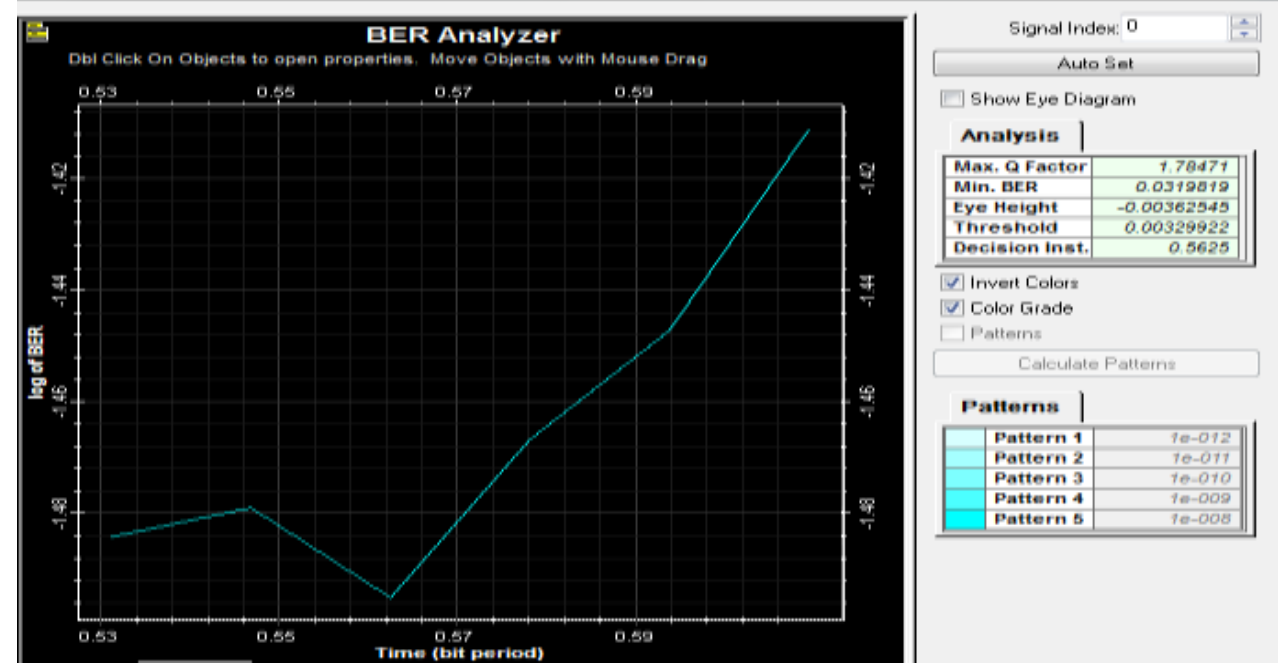

Figure 5. Min. data error rate value estimation based on the DPIM transmission coder
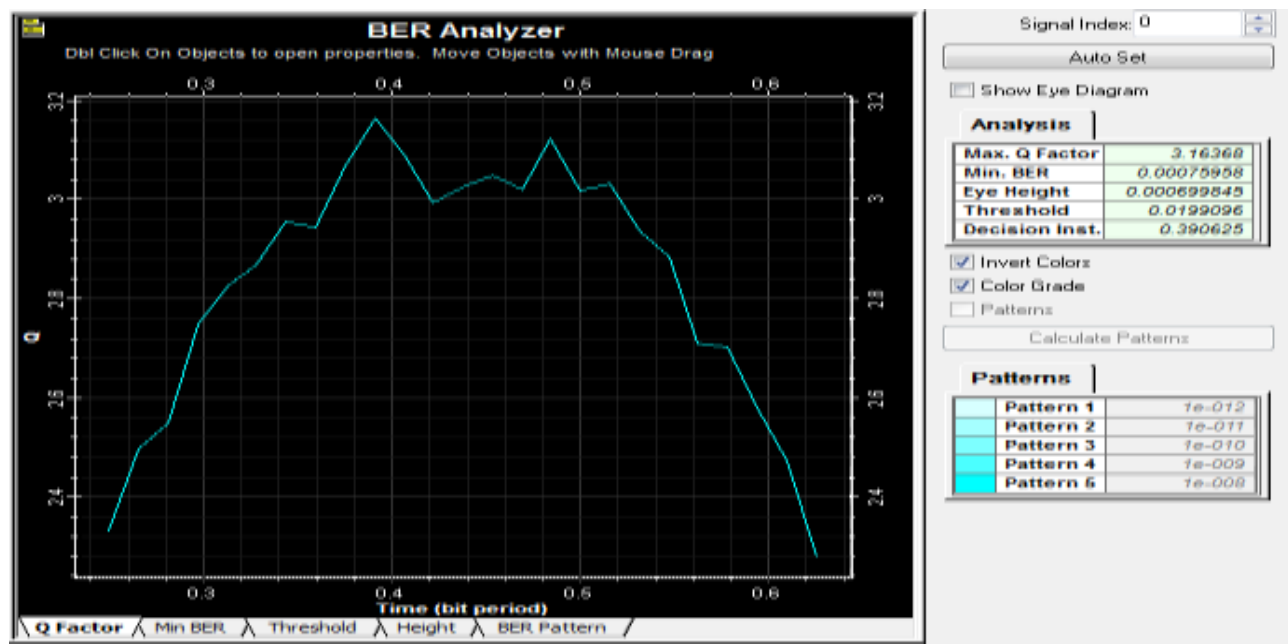

Figure 6. Max. Q parameter value estimation based on the 4B5B modulation transmission coder
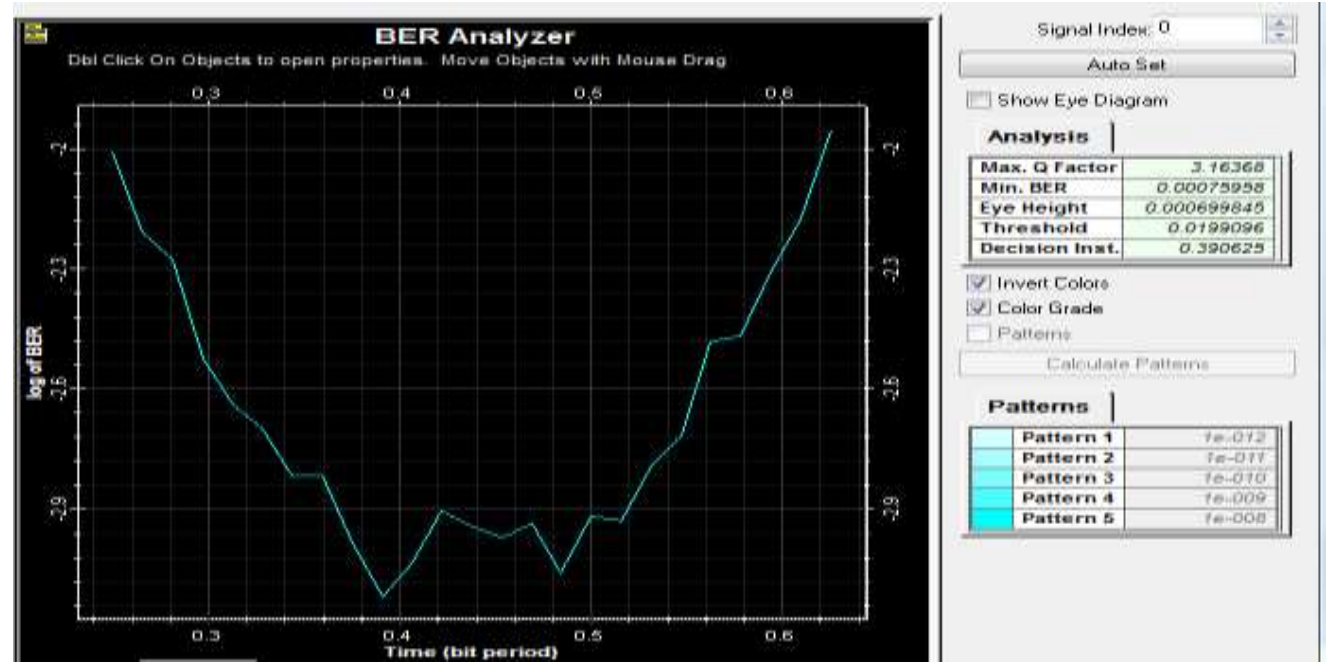

Figure 7. Min. data error rate value estimation based on the 4B5B modulation transmission coder 


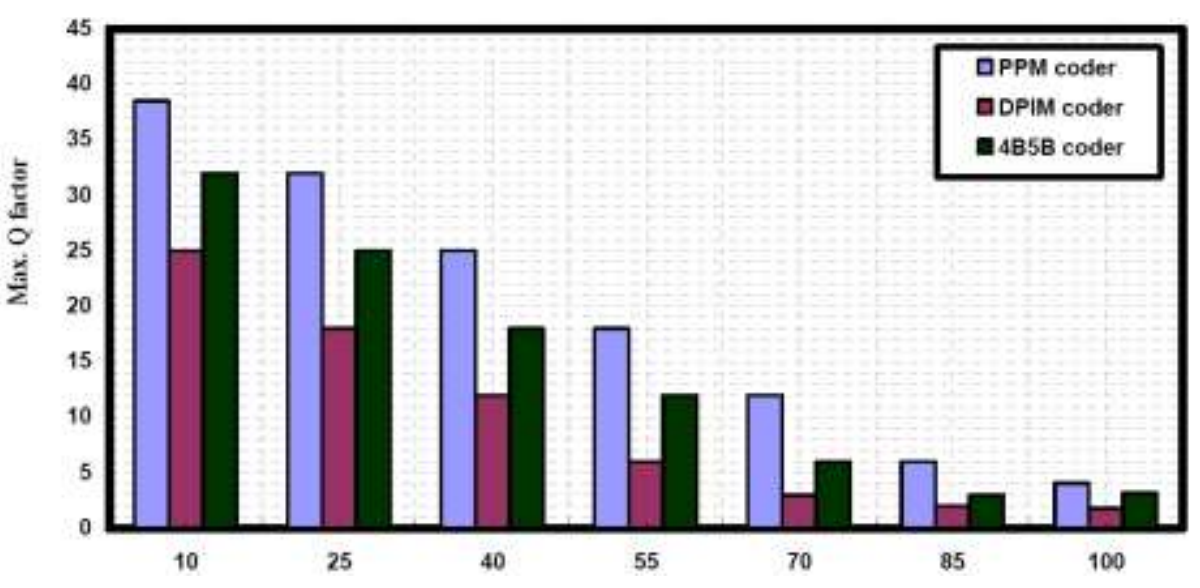

Figure 8. Maxim Q coefficient variations versus propagation distance variations for various transmission coders

\section{CONCLUSION}

In summary, the laser-measured rate equations with various transmission coders are simulated to ensure optimal data transmission error rates. Different transmission pulse coders are employed to enhance the system performance efficiency. It can be observed that the pulse position modulation coder has presented a $17.65 \%$ higher enhancement percentage ratio in its Q-value than the 4B5B modulation transmission coder. The PPM transmission coder also outlined a $36.98 \%$ higher enhancement percentage ratio in its Q-value than the DPIM transmission coder. Therefore, it can be concluded that the PPM transmission coder is the best candidate for a transmission pulse coder for high data rate transmission in fiber-optic systems.

\section{REFERENCES}

[1] Ahmed Nabih Zaki Rashed, "Optical Network Management and Its Performance Evaluation for Both Future Cost Planning and Triple Play Solutions," Wireless Personal Communications Journal, Springer Publisher, vol. 75, no.4, pp. 2005-2020, April 2014.

[2] Ahmed Nabih Zaki Rashed, Mohamed A. Metwae'e, "Optical Filters Dimensions and Thermal Operation Conditions Impact on Its Transmission Considerations in Near Infrared (NIR) Optical Spectrum Transmission Region," Optoelectronics and Advanced Materials Journal-Rapid Communications, vol. 8, no. 3-4, p. 175-184, March-April 2014.

[3] Christopher J. Stapels, et al., "CMOS Based Avalanche Photodiodes for Direct Particle Detection," Nuclear Instruments and Methods in Physics Research A, vol. 579, no. 1, pp. 94-98, Aug. 2007.

[4] A. Pulvirenti, et al., "Characterization of Avalanche Photodiodes (APDs) for the Electromagnetic Calorimeter in the ALICE Experiment," Nuclear Instruments and Methods in Physics Research A, vol. 596, no. 1, pp. 122-125, Oct. 2008.

[5] S. Naghizade, S. M. Sattari-Esfahlan, "Excellent Quality Factor Ultra-Compact Optical Communication Filter on Ring-Shaped Cavity," Journal of Optical Communications, vol. 40, no. 1, pp. 21-25, Jan. 2019.

[6] Y. Hou, H. Tao, F. Hou, "Modified Periodic Encoder for Increasing Pulse Height in PON Monitoring System," Journal of Optical Communications, vol. 40, no. 1, pp. 27-30, Jan. 2019.

[7] A. Yousuf, Najeeb-Ud-Din Hakim "Investigation on Chirping induced performance degradation in Single Mode Directly modulated 1.55um DFB laser," Journal of Optical Communications, vol. 40, no. 1, pp. 31-36, Jan. 2017.

[8] C.-C. Kao, et al., "The Lasing Characteristics of GaN Based Vertical Cavity Surface Emitting Laser With AlN-GaN and $\mathrm{Ta}_{2} \mathrm{O}_{5}-\mathrm{SiO}_{2}$ Distributed Bragg Reflectors," IEEE Photon. Technol. Lett., vol. 18, no. 7, pp. 877-879, 2006.

[9] S.-K. Wang, et al., "Optically Pumped GaN Based Vertical Cavity Surface Emitting Lasers: Technology and Characteristics,” Jpn. J. Appl. Phys., vol. 46, no. 8B, pp. 5397-5407, 2007.

[10] T.-C. Lu, et al., "CW Lasing of Current Injection Blue GaN Based Vertical Cavity Surface Emitting Laser," Appl. Phys. Lett., vol. 92, no. 14, pp. 1-3, 2008.

[11] Ahmed Nabih Zaki Rashed, et al., "Transmittivity/Reflectivity, Bandwidth, and Ripple Factor Level Measurement for Different Refractive Index Fiber Grating Shape Profiles," Journal of Optical Communications, Published Online: 6 Feb. 2019, https://doi.org/10.1515/joc-2018-0233.

[12] Ahmed Nabih Zaki Rashed, et al., "DWDM Channel Spacing Effects on the Signal Quality for DWDM/CWDM FTTx Network," Journal of Optical Communications, Published Online: 7 Feb. 2019, https://doi.org/10.1515/joc2019-0012. 
[13] Ahmed Nabih Zaki Rashed, et al., "Numerical Analysis of Optical Properties Using Octagonal Shaped Photonic Crystal Fiber," Journal of Optical Communications, Published Online: 8 Feb. 2019, https://doi.org/10.1515/joc2019-0013.

[14] Ahmed Nabih Zaki Rashed, et al., "Different Graded Refractive Index Fiber Profiles Design for the Control of Losses and Dispersion Effects," Journal of Optical Communications, Published Online: 19 Feb. 2019, https://doi.org/10.1515/joc-2019-0036.

[15] Ahmed Nabih Zaki Rashed, "Comparison between NRZ/RZ Modulation Techniques for Upgrading Long Haul Optical Wireless Communication Systems," Journal of Optical Communications, Published Online: 19 Feb. 2019, https://doi.org/10.1515/joc-2019-0038.

[16] IS Amiri, et al., "Interaction between Optical Sources and Optical Modulators for High-Speed Optical Communication Networks," Journal of Optical Communications, Published Online: 15 March 2019, https://doi.org/10.1515/joc-2019-0041.

[17] IS Amiri, et al., "Effects of Order Super Gaussian Pulses on the Performance of High Data Rate Optical Fiber Channel in the Presence of Self Phase Modulation," Journal of Optical Communications, Published Online: 5 April 2019, https://doi.org/10.1515/joc-2019-0039.

[18] IS Amiri, et al., "Mathematical Model Analysis of Dispersion and Loss in Photonic Crystal Fibers," Journal of Optical Communications, Published Online: 5 April 2019, https://doi.org/10.1515/joc-2019-0052.

[19] IS Amiri, et al., "Basic Functions of Fiber Bragg Grating Effects on the Optical Fiber Systems Performance Efficiency," Journal of Optical Communications, vol. 0, issue 0, Published Online: 5 April 2019, https://doi.org/10.1515/joc-2019-0042.

[20] IS Amiri, et al., "Nonlinear Effects with Semiconductor Optical Amplifiers," Journal of Optical Communications, Published Online: 12 April 2019, https://doi.org/10.1515/joc-2019-0053.

[21] IS Amiri, et al., "High-Speed Light Sources in High-Speed Optical Passive Local Area Communication Networks," Journal of Optical Communications, vol. 0, issue 0, Published Online: 20 April 2019, https://doi.org/10.1515/joc2019-0070.

[22] IS Amiri, et al., "Spatial Continuous Wave Laser and Spatiotemporal VCSEL for High-Speed Long Haul Optical Wireless Communication Channels," Journal of Optical Communications, Published Online: 24 April 2019, https://doi.org/10.1515/joc-2019-0061.

[23] IS Amiri, et al., "Average Power Model of Optical Raman Amplifiers Based on Frequency Spacing and Amplifier Section Stage Optimization," Journal of Optical Communications, vol. 0, issue 0, Published Online: 4 May 2019, https://doi.org/10.1515/joc-2019-0081.

[24] IS Amiri, et al., "Temperature effects on characteristics and performance of near-infrared wide bandwidth for different avalanche photodiodes structures," Results in Physics, vol. 14, September 2019, https://doi.org/10.1016/j.rinp.2019.102399.

[25] IS Amiri, Ahmed Nabih Zaki Rashed, "Simulative study of simple ring resonator-based brewster plate for power system operation stability," Indonesian Journal of Electrical Engineering and Computer Science, vol. 16, no. 2, pp. 1070-1076, November 2019, DOI: 10.11591/ijeecs.v16.i2.pp1070-1076.

[26] IS Amiri, et al., "Comparative Simulation Study of Multi Stage Hybrid All Optical Fiber Amplifiers in Optical Communications," Journal of Optical Communications, Published Online: 4 Feb. 2020, https://doi.org/10.1515/joc2019-0132.

[27] IS Amiri, et al., "Optical Communication Transmission Systems Improvement Based on Chromatic and Polarization Mode Dispersion Compensation Simulation Management," Optik Journal, vol. 207, April 2020, https://doi.org/10.1016/j.ijleo.2019.163853.

[28] Ahmed Nabih Zaki Rashed, et al., "Distributed Feedback Laser (DFB) for Signal Power Amplitude Level Improvement in Long Spectral Band," Journal of Optical Communications, vol. 0, issue 0, Published Online: 2 April 2020, https://doi.org/10.1515/joc-2019-0252.

[29] IS Amiri, et al., "Analytical Model Analysis of Reflection/Transmission Characteristics of Long-Period Fiber Bragg Grating (LPFBG) by Using Coupled Mode Theory," Journal of Optical Communications, Published Online: 2 April 2020, https://doi.org/10.1515/joc-2019-0187.

[30] IS Amiri, et al., "Conventional/Phase Shift Dual Drive Mach-Zehnder Modulation Measured Type Based Radio over Fiber Systems," Journal of Optical Communications, Published Online: 14 April 2020, https://doi.org/10.1515/joc-2019-0312.

\section{BIOGRAPHIES OF AUTHORS}

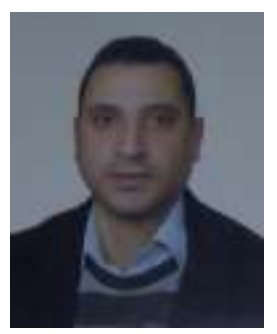

Dr. Hazem M. El-Hageen is currently an assistance professor of Electrical Engineering Department at the Tabuk University. He also worked for Egyptian Atomic Energy Authority and Nuclear \& Radiological Regulatory Authority. He received the B.Sc., M.Sc., and Ph.D scientific degrees in the Electronics and Electrical Communication Engineering Department from Faculty of Electronic Engineering, Menoufia University in 2000, 2005, and 2012 respectively. His research mainly focuses on Active and Passive Optical Networks. His research interests include Optical Communication Systems, Optoelectronics Devices, Optical Fiber, Filters and Sensors. Other areas of interest are Ionizing Radiation Effects in Solid State Materials and Devices, Digital Communication, Embedded, Control and Automotive Systems. First Author affliction is 
Electrical Engineering Department, Faculty of Engineering, University of Tabuk, Tabuk, Saudi Arabia. Second Author affliction is Egyptian Nuclear \& Radiological Regulatory Authority, Cairo, Egypt. His email: hazemhageen@hotmail.com.

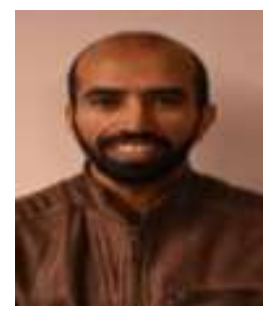

Dr. Aadel Alatwi was born in Tabuk, Saudi Arabia, in 1980. He received the B.S. degree from King Abdul-Aziz University, Jeddah, Saudi Arabia, in 2004, the M.S. and Ph.D degrees from Griffith University, Brisbane, Australia, in 2008 and 2018 respectively, both in communication engineering. He is currently assistant professor in the School of Engineering at Tabuk University, Tabuk, Saudi Arabia. His current research interests include speech coding, speech and speaker recognition, speech enhancement, face recognition, image coding, pattern recognition and artificial neural networks. His email: aadel.alatwi@ut.edu.sa

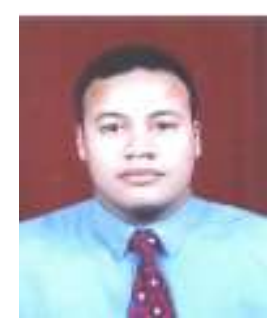

Assoc. Prof. Ahmed Nabih Zaki Rashed was born in Menouf city, Menoufia State, Egypt country in 23 July 1976. Received the B.Sc., M.Sc., and Ph.D. scientific degrees in the Electronics and Electrical Communications Engineering Department from Faculty of Electronic Engineering, Menoufia University in 1999, 2005, and 2010 respectively. Currently, his job carrier is a scientific lecturer in Electronics and Electrical Communications Engineering Department, Faculty of Electronic Engineering, Menoufia university, Menouf. Postal Menouf city code: 32951 , EGYPT. His scientific master science thesis has focused on polymer fibers in optical access communication systems. Moreover, his scientific Ph. D. thesis has focused on recent applications in linear or nonlinear passive or active in optical networks. His interesting research mainly focuses on transmission capacity, a data rate product and long transmission distances of passive and active optical communication networks, wireless communication, radio over fiber communication systems, and optical network security and management. He has published more than 227 published scientific papers in international journals and conferences.

He has published many high scientific research papers in high quality and technical international journals in the field of advanced communication systems, optoelectronic devices, and passive optical access communication networks. His areas of interest and experience in optical communication systems advanced optical communication networks, wireless optical access networks, analog communication systems, optical filters and Sensors. As well as he is editorial board member in high academic scientific International research Journals. Moreover, he is a reviewer member in high impact scientific research international journals in the field of electronics, electrical communication systems, optoelectronics, information technology and advanced optical communication systems and networks. His personal electronic mail ID (Email:ahmed_733@yahoo.com). His published paper under the title "High reliability optical interconnections for short range applications in high performance optical communication systems" in Optics and Laser Technology, Elsevier Publisher has achieved most popular download articles in 2013. 